\title{
The Anatomy of The Fabela Bone and its Clinical Importance
}

\author{
Sóstenes S. Oliveira, ${ }^{1}$ Wellington J. S. F. Oliveira ${ }^{2}$
}

${ }^{1}$ Department of Morphology, Federal University of Rio Grande do Norte, Natal-RN, Brazil

${ }^{2}$ Department of Physiotherapy, Faculty Mauricio de Nassau, Natal-RN, Brazil

Disclose and conflicts of interest: none to be declared by all authors

\begin{abstract}
Introduction: the fabella bone is characterized as a sesamoid that is located in the posterior region of the knee, precisely in the tendon of the lateral head of the gastrocnemius muscle, behind the lateral femoral condyle. During routine physical therapy visits, pain was found in the functional evaluation of the posterior region of the knee during flexion-extension movements of the right knee. A radiograph of the right knee in the lateral plane was requested and the fabela bone was evidenced. Patients with fabella may be asymptomatic. However, here we find a case in which the fabela bone may be associated with pain in the lateral aspect of the knee.
\end{abstract}

Keywords: Human anatomy; Knee pain; Fabela.

\section{Introduction}

The fabela bone is characterized as a sesamoidtype bone that is located in the posterior region of the knee, precisely in the tendon of the lateral head of the gastrocnemius muscle, behind the lateral femoral condyle ${ }^{1}$.

Fabellar Syndrome (SF) is related to knee pain, synovial irritation and degeneration of the joint surface. The presence of fabella can be examined by palpation or by complementary exams, such as radiographs, ultrasounds and magnetic resonance. However, Fabela is very neglected by many professionals and can be one of the factors for such errors in clinical and kineticfunctional diagnoses ${ }^{2}$.

\section{Case Report}

A 62-year-old patient presented to the Physiotherapy Department of the Unified Health System in the city of Bento Fernandes, Rio Grande do Norte, Brazil, with a history of knee pain, specifically in the posterolateral compartment of the knee after being diagnosed with osteoarthritis on the right knee. There was no history of knee problems previously. On physical examination, she presented joint effusion and pain on palpation in the popliteal region, but no muscle defect was noted.

During the functional evaluation, crackling sounds were noticed during the knee flexion-extension movements, reporting painful signs at $40^{\circ}$ of knee flexion. During the entire knee extension movement, no pain symptoms were noted. She reports that she feels a lot of pain when climbing stairs and performing the movements of sitting and getting up from a chair, for example.

The examination requested by the physiotherapist was an X-ray of the right knee in the sagittal plane with lateral view. Upon receipt of the report, the diagnosis of arthrosis in the knee joint was closed. However, in the evaluation of the radiography, now, being more careful, the fabela bone was noticed by the physiotherapist (Figure 1).

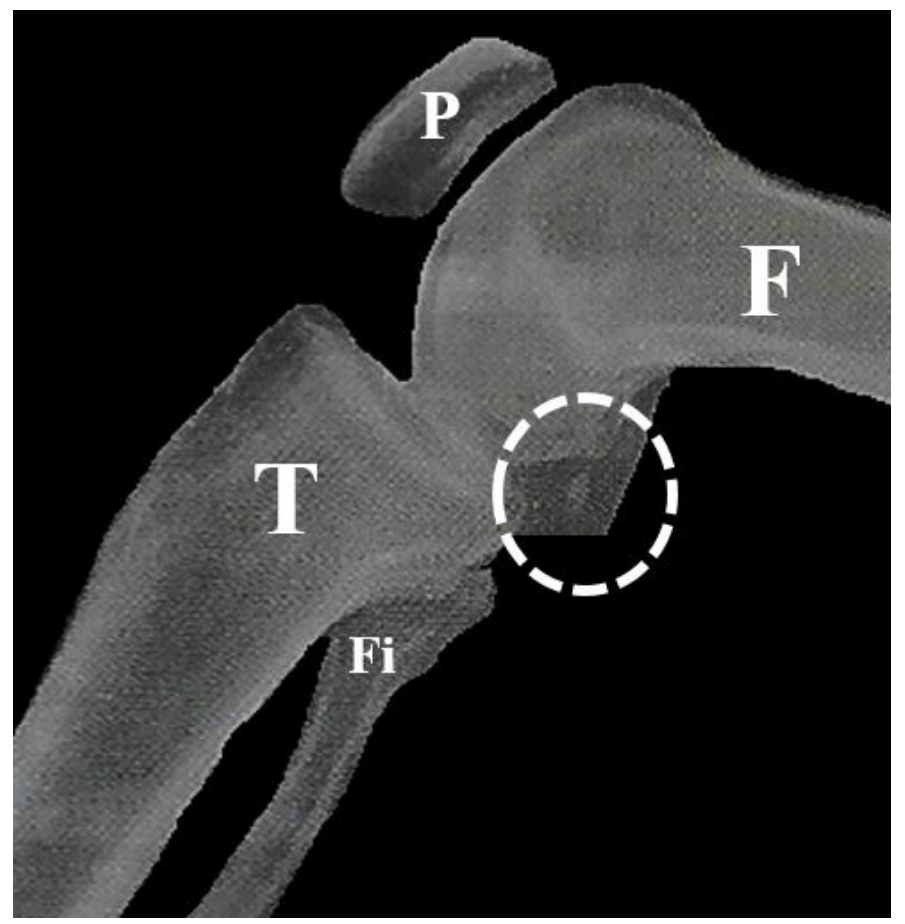

Figure 1. Radiograph of the right knee. F: femur; P: Patella; T: Tibia; Fi: fibula; White circle in strokes: Bone fabela

\section{Discussion}

The case report published here supports that fabela bone can be detected in routine radiographic examinations and that it will lead to a more careful search for physiotherapeutic approaches to be performed on the patient in order to reduce the pain, improve the range of motion and increase the strength 
of the limb without feeling pain at the moment of the evaluated movements and that will be used in daily life.

Fabela bone is present more in men than in women and the fabela prevalence rate increases with age, they can ossify in the beginning of life, at 12 years old, or later, until the mid 70s. In most cases the fabela is present on both knees, however, in the unilateral cases of the fabela, it is present more on the right knee. We can say that it is present on average $36.8 \%$ of the knees worldwide, being more prevalent in the population of Asia, Oceania and South America than in Europe, the Middle East, North America and Africa ${ }^{3}$.

It is worth noting that the fabela is more evident in studies that use cadaveric dissection as a method than for those based on imaging tests, for example radiography. This is due to the fact that studies with dissections of the fabela bone may be presented in a more cartilaginous or ossified form, but less dense ${ }^{1,3}$. Here in this case studied, we have more evidence that the fabella can be seen in routine radiographic examinations.
We do not have many studies in the scientific literature that have investigated the function of fabella ${ }^{3}$. Some studies have described that the fabela may be related to the development of the fabelofibular ligament ${ }^{4}$, which may help to improve the stability of the posterolateral knee complex ${ }^{5}$ and finally suggested until then that the fabela may be acting as an advantage mechanics for the inserted muscle ${ }^{6}$.

\section{Conclusion}

The knowledge on the part of health professionals of the existence of fabela bone is of great importance for the daily practice of the professional who performs the activities in the operating room or in the outpatient clinic. The fabella can trigger SF and cause pain in the posterolateral region of the knee. However, there are several types of diagnosis that can be considered during the evaluation of the patient who presents the fabela bone, because at the time of the evaluation, a more careful evaluation that will designate a more specific conduct for these cases is extremely important.

\section{References}

1. Adukia V, Ricks M, Colquhoun K, Flynn N (2019). An anomalous band originating from the fabella causing semimembranosus impingement presenting as knee pain: a case report. Journal of Medical Case Reports. 13 (1): 1-4.

2. POP, T. S., POP, A. M., OLAH, P., AND TRÂMBITASS, C (2018). Prevalence of the fabella and its association with pain in the posterolateral corner of the knee: A cross- sectional study in a Romanian population. Medicine, 97, 47.

3. Berthaume MA, BULL AMJ (2020). Human biological variation in sesamoid bone prevalence: the curious case of the fabella. Journal of anatomy. 236 (2): 228-242.
4. Minowa, Takeshi, et al (2004) .Does the fabella contribute to the reinforcement of the posterolateral corner of the knee by inducing the development of associated ligaments ?. Journal of Orthopedic Science 9 (1): 59-65.

5. Eyal, Shai, et al (2019). Common cellular origin and diverging developmental programs for different sesamoid bones. Development 146.4 .

6. Driessen, Arne, et al (2014). The fabella syndrome-a rare cause of posterolateral knee pain: a review of the literature and two case reports. BMC Musculoskeletal Disorders 15.1: 100.
Received: February 4, 2021

Accepted: March 27, 2021
Corresponding author

Sóstenes Silva de Oliveira

E-mail: sostenesfisio@gmail.com 